\title{
Narrating Autism: Autistic Presence and Voice on the internet in Indonesia
}

\author{
Hersinta \\ STIKOM LSPR Jakarta, Mass Communication Dept, Jakarta, Indonesia \\ Curtin University, School of Media, Creative Arts and Social Inquiry, Western Australia
}

$\{$ hersinta@1spr.edu\}

\begin{abstract}
Autism, is a relatively 'current' concept in Indonesia, with the public awareness about autism in Indonesia only began emerging in 2000 and was particularly prevalent through online media. Today, the internet has become a recognized medium for self-narrative and self-expression for this group. Autistic people have participated online in a number of formats including autobiographical blogs, video awareness campaigns on YouTube, and through efforts to build online communities by utilizing social media. This paper will discuss the rise of autistic online presence in Indonesia by conducting in-depth interviews with five autistic persons in Indonesia. Data drawn from the interviews investigates the main questions for this research: (1) how they utilize social media to provide a self-narrative and as a tool for doing self-advocacy; and (2) how the internet could provide different ways of understanding autism. Results indicate that social media acts as an embedded media for autistics, as they are using it extensively in daily activities, as well as a mediated tool for self-advocating and communicating their narratives.
\end{abstract}

Keywords: Autism; Disability; Internet; Neurodiversity; Online Self-Advocacy

\section{Introduction}

The public history of autism in Indonesia has just started in the last two decades, with limited amount of academic research in that area [1]. Considering the fact that autism is a relatively current topic in Indonesia and the limitation of access to media and health information in some areas, a large percentage of Indonesians still have little understanding of autism, particularly for those who live in rural and remote areas [2,3]. Furthermore, there is still no exact data on the rate of autism prevalence from reliable sources, although according to the Ministry of Health reports, there were 475,000 children diagnosed with autism in 2004 and the number of children diagnosed with the disorder is continuing to increase [2].

As stated by the American Psychiatric Association (APA), people with autism have impairments related to communication and social interaction, and might have the presence of unusual or repetitive behavior or language, delay of speech or lack of creative pretend play [4,5]. First identified by Leo Kanner in 1943 as a childhood syndrome characterized by "autistic aloneness" [6], the degrees of severity within the autism spectrum are varied, ranging from mild to severe. There is a distinction between mild or "high-functioning autists" - widely 
known as those with Asperger syndrome, referring to autistic individuals who have "an IQ in the normal range or above" - and "low-functioning autists" [6]. But this distinction is arguable, as some previous research and literatures found that autistics who considered nonverbal or having limited speech (or classified as low functioning) could achieve high score on the nonverbal assessment of intelligence and could communicate through digital technology $[5,7]$.

Social media use among autistics has been one of the interesting topics to explore, as researchers paid more attention to the impacts of its use, particularly by people at the highfunctioning end of the spectrum [4,8,9]. Social media and other internet-based communication- such as blogs, email and online forums can provide "a highly structured environment without extraneous stimuli" [10]. With its visual anonymity, more flexible pace of communication and prominence of text, the internet can facilitate communication needs and break down some of the social barriers experienced by autistic people, thus allowing them to: (1) acquire greater control over social communication; (2) have contact with others facilitated by the technology; (3) share their stories with the support of the digital platform [8,11]. In the global context, driven by the use of computers and the development of communications technology through the internet and new media, the online autism community serves as a communication platform and also an advocacy network for people with high functioning autism and Asperger syndrome [7,11,12].

Recently some autistic individuals in Indonesia started to speak out about their experiences of being a person on the spectrum through online platforms. Online personal narratives, often in the form of blogs, also allow autistic individuals to voice their identities and increase awareness on the neurodiversity discourse [13]. For example, blog was largely chosen by disability activists to advocate for disability issues and "antithesis to what the mainstream media is presenting" for the media often portray people with disability either as the "supercrip (the hero)" or the "lazy crip (the lowlife)" [14]. In Indonesia, Oscar Dompas, an autistic man, utilized a website for keeping journals of his daily life. Oscar's website was first initiated by his father, Jeffrey Dompas, who wanted to make an outlet for his son for writing his daily life experiences [15]. Those examples showed changes- although still exiguous- in autism advocacy in Indonesia over the past decade. It is worth to note that the movement firstly initiated by parents and professionals dealing with autistic children - who mainly implied the medical approach by viewing autism as a disorder that needs to be cured. Subsequently, it seems to be evolving in the form of the growing presence of autistic individuals [13]. This group identified themselves as person with differences, not as person with disorders- which more applies to the social model of disability. Interestingly, by utilizing the internet and social media as the medium, these person with autism currently start to communicate their presence by sharing their experiences of being autistic and doing advocacy on behalf of themselves. Using the online media platform, they cast a new understanding about autism as another human difference; one not to be treated as a disorder, but as another human specificity such as gender or sexuality [13].

\section{Method}

For the purpose of this study, qualitative research methods were applied by conducting data collecting in the form of semi-structured interviews and observations of social media contents created by autistics. A set of interview guide consists of open-ended questions about participants' experiences and perceptions related to social media use and autism advocacy, as 
well as on understanding autism and identity was used during the interviews. The interview settings were done using online and offline communication modes- either in synchronous such as chat or asynchronous as through email as well as through face-to-face interview- depends on the convenience of the participants. Participation was voluntary and ethical approval for this study was obtained through appropriate channels at Curtin University, Western Australia.

To explore further of this minority voice- the Indonesian autistic people who utilize social media to provide a different discourse on autism- five participants were selected to be interviewed. Prior to the interview, a content analysis using textual and social data analytics software (DiscoverText) on Twitter was done to map the conversations and to observe active accounts about autism and the neurodiversity movement in social media in Indonesia. Based on the results, three active users who self-identified themselves as the people on the spectrum were recruited as participants: (1) AN, an Aspie person or a person who has Asperger Syndrome; (2) YR, who identified herself as autistic and posted tweets about neurodiversity; (3) FG, who published an article on self-experience as autistic person in an Indonesian-based online publication focusing on women and gender issues. Besides these three participants, two more participants representing the same category- as people on the spectrum and discussing autism and advocacy online- were also recruited. These include (4) LA, who once initiated Indonesian Autism Youth on Twitter and (5) FY, an autistic youngster who is actively using social media. Despite the fact that all participants have been diagnosed with autism spectrum or Asperger Syndrome (AS), they have a different age range (from 19 to 51), occupations, and a variety of conditions.

\section{Results and Discussion}

In this part, the results and discussions will be described in three parts drawn from the participants' responses from the interviews, which include: social media use among autistics, online autistic self-advocacy and how autism is perceived in Indonesia.

\subsection{Social Media Use Among Autistics}

According to all participants, social media usage is part of their daily essential activities. For example, FG uses various kind of social media and online messaging platforms in her daily activities, such as to find interesting posts, connecting with friends, and interactive communication or chatting. Similar preference came from FY who mentions Instagram for browsing on her favorite things and WhatsApp for daily communication and social interaction. Another informant, AN, stated he also utilizes social media- such as Instagram and Facebookfor promotional use to support his work as a musician and his role as a 'social media influencer'. Other participants also make use of social media for work and professional use, such as LA and YR who use Tumblr for offering their services as digital artists. As described by LA, she uses social media every day. According to her, social interaction is the most important thing because she often has difficulties to interact with people in real life. For all participants, social media is regarded as an 'embedded media' - a term that Philip Howard [6] refers to people's increasing reliance on the communication technology in their daily activities. Despite that, social media also served as comfortable mediated tools for facilitating social interactions among participants, as well as for communicating their views and looking for information sources. 
For most participants, written communication form seems to be their preferred mode of communication. As an example, YR said that in oral communication, it seems that she needs "longer time from the brain to mouth. But with typing, I don't have to move my mouth, so it's faster". Other participants like LA also writes in her blog that she prefers typing when communicating, "as it is a great technique for people who can imagine word with its structure, but have difficulties to use their mouth to explain". Talking and communicating via the textual form and through social media were viewed as more comfortable way and straightforward for doing social interaction by most of the participants. As YR and FG explained, there are fewer distractions when communicating via the internet and also lessen the anxieties of misinterpreting the non-verbal cues. Although there are exceptions- if the conversation involves very close friends or relatives, some of the participants, would prefer face-to-face communication over social media conversation, as "it is usually more dynamic". Davidson [17,18] argued that the written word is preferred among autistics for self-expression and communicative interaction due to their experiences of having repeated stress or 'meltdown' situations in processing environmental stimuli. Previous study by Penny and Standen [8] acknowledged that time flexibility in online communication can provide "more control over the structure of conversations in terms of pace, topic and turn-taking". Those arguments from previous studies show good congruence with participants' experiences in this study. Moreover, using mediated tools like the internet for communication and social interaction can lessen the distractions for people with Asperger Syndrome and "high-function" autism, who have difficulties in nonverbal aspects of social communication, such as interpreting gestures, facial expressions and maintaining eye contacts [8].

\subsection{Online Autistic Self-Advocacy}

All participants were engaged in online autistic self-advocacy in various forms. AN uses social media for advocacy and to raise awareness about Asperger's and autism issues using the hashtag \#AspieIsAwesome in twitter and posts a YouTube link in which he was being interviewed about his experience as a person on the spectrum. Regarding his role as an advocate for Asperger's and autism issues in social media, AN views it has some positive impacts: "People know more about the issues, and I got positive responses from them. One of them actually contacted me and currently, we are working for a book project about Asperger's. The benefits (of advocacy in social media) actually is more for other people. For me, well, I'd rather say "I have this (condition)." LA first started to blog under the name of "Indonesian Autism Youth" or PAI in 2014, she also speaks critically on some issues relating to autism through her Twitter personal account and YouTube videos she created. FY, regularly writes in her two blogs- in Kompasiana and Blogspot- about autism topics and her daily activities and experiences. Her blog in Kompasiana consists more of autism-theme articles, ranging from coverage of autism events to interviews with other autistic teens and other people about autism issues, while her articles in Blogspot are more like her daily journal.

Writing an online article also preferred by FG to share her experience of being a person on the spectrum. She published an article titled "Coming Out as Autistic" in Magdalene, an online publication in Indonesian gender and cultural issues. While another participant, YR, often used Twitter and Tumblr for starting a thread or involving in conversations related to autism. Interestingly, she also finds that social media serves as a resource "to have their words heard" because in the 'real' world such spaces are reserved mainly by the parents and health practitioners. That is why she does not prefer to search for autistic resources online, and more inclined towards a discussion with particular online friends who are on the same spectrum. 
In terms of autism advocacy - both online and offline - in Indonesia autism advocacy is mainly initiated by parents of autistic children, such as the Autism Foundation of Indonesia (YAI), the Indonesian Autism Awareness Community (MPATI), and an online mailing list forum called Putra Kembara. These pioneering advocacy groups and online forum were already started in the late 1990s and the beginning of 2000s, coinciding with the time when Indonesia starting to "more fully embrace the popularity of the internet and the power of online communication" [1]. However, it is worth to note that in order to establish a selfadvocacy disability movement, there are two important keys: self-help skills and group organizing [12]. The first is regarded as an important matter to gain one's dependency, as people can manage to take care of their own. The second refers to finding allies based on the "group identity", that people with disabilities are having the same social experiences with each other, despite they are having a similar or different type of disabilities [12]. These two things, apparently, are still lacking in Indonesia. Firstly, most of the autistics (at least the ones who represented in the news and featured by the Indonesian pioneering autism organizations) in Indonesia are still on the process to gain their independence and acquire self-help skills. Secondly, the autistic self-advocacy in Indonesia is still based on personal identity, not on a group identity where they can share the same social identities to each other. While until currently there is no autistic organization (both online and offline) on behalf of autistic people in Indonesia, some parents-based autism organizations already took further steps in making some collaborations with autistic youngsters to support their autism advocacy movement and to provide opportunities for autistics to participate in the larger society.

\subsection{How Autism is Perceived in Indonesia}

In Indonesia, autism is identified under the category of mental disability according to the Ministry of Health [19], although autism is put on different categories by different government bodies. Even in the current situation, most of the participants also view that Indonesian people still have limited understanding of what autism is. Two of participants have different opinions about how autism is perceived in Indonesia. YR, for example, has a bleak opinion on how autism is still seen "as an illness, sadly- no matter what the country is- instead of a different way for our brain to work." However, FY mentioned a different experience as she views that other people will understand her condition and look at her as 'normal' after she explained about her condition.

While all participants have similar views on understanding autism as a difference, not all of them are familiar with the term 'neurodiversity' or 'neuro diverse' as depicting autism as a part of neurological differences among humans. The term itself is not widely used in an Indonesian context, either in the media and in the advocacy movements- as the first one tends to apply a medical approach and the latter is still largely dominated by parents-based organization movements. The neurodiversity view is predominantly disseminated through the online platforms and particular communities outside Indonesia, including in non-Indonesian autistic self-advocacy groups and online fandom discussions related to autism characters. Despite using the term 'neurodiverse', Indonesians are generally more familiar with the term 'special needs', although the this term is considered as a disability euphemism and not a legal term in another countries, such as in the United States [20].

Apart from that, in Indonesia, there is also misunderstanding on how people use the term "autis" (autist) for referring to people who are immersed deeply with their gadgets. This situation was acknowledged by all participants who express their concern on how it trivializes people's understanding of autism in Indonesia. As LA said, "It is pretty sad how it trivializes 
autism and how it does not make Indonesians aware of autism and how to accommodate them from all groups in Indonesia." The fact that autism is a broad spectrum with various conditions and ability levels, ranging from profound mental retardation to superior intelligence [21] also makes it more difficult to understand for a general audience.

Despite people's unfamiliarity of the neurodiversity term in Indonesia, there is also a stigma surrounding mental issues and disabilities as stated by some participants. In YR's opinion, for example, she states that a person who experienced mental breakdown condition still being regarded as 'possessed by something (such as an evil spirit)'. While LA views that mental health is not taken as seriously in Indonesia as it is in the West. She perceives in Indonesia mental illness is associated with people on the streets, so it will be challenging to understand autism as a part of neurodevelopmental disorders.

In some areas in Indonesia, traditional and cultural beliefs such as karma and taboo still permeate people's understandings about disability, including autism, which can lead to stigmatization and discrimination toward disabled people. In some areas in Indonesia, traditional and cultural beliefs such as karma and taboo still permeate people's understandings about disability, including autism, which can lead to stigmatization and discrimination toward disabled people. Regarding to the diverse understanding and perception about autism among different groups (and classes) in Indonesia, the answer probably relates to how autism (and disability) is socially constructed and culturally represented in different settings. Kim's study [22] in social implications of autism in different countries- Korea, Nicaragua, and Canadashows that cultural perspective could pervade people's perceptions of autism and disability. In Korea, for example, cultural factors such as patriarchal traditions may force mothers to be blamed for autism- thus force acceptance of another diagnosis (RAD or Reactive Attachment Disorder) instead of being diagnosed with autism. In some urban areas in Indonesia, Tucker [1] found that autism has less social stigma compared to psychotic disorder, as parents prefer 'autism' diagnosis rather than schizophrenia diagnosis.

\section{Conclusion}

Based on the results and discussion, it could be seen that, firstly, social media acts as an embedded media for autistics, as they are using it extensively in daily activities. Ranging from social interaction, promotional or marketing use, getting in touch with families and peers, seeking references, to voicing out their opinions and sharing their stories. Those activities were facilitated by using the online communication technology. Secondly, social media has become a mediated tool for their presence and advocating for themselves. Social media such as Twitter, Tumblr, blog, Instagram and YouTube are utilized as an alternative platform for them to speak on behalf of autistics. These online platforms also become the medium for autistics to get information, and provide different understandings about autism-related to neurodiversity approach for some participants.

Thirdly, while all the participants have similar views on understanding autism as a difference, they also acknowledge that in Indonesian people have limited understanding on autism, possibly among the lower class group. Presumably, it is related to the historical aspect that autism is cultural representations of disability and traditional beliefs have large impacts on people understanding about autism. In Indonesia, autism is still related to karma and taboo, or labeled as being odd or mad, or possessed by an evil spirit. These can lead to stigma and discrimination toward autistic people, adding to the impact of autism as a non-physical or 'invisible disability'. As a final note, in discussing autism online presence and self-advocacy 
in Indonesia, it is important to acknowledge that the autistic self-advocacy is still based on personal identity, not on a group identity where they can share the same social identities to each other. Up to this time on conducting this study, there is no autistic organization (both online and offline) exists yet despite parents-based autism organizations. Having said that, these organizations currently made some collaborations with autistic youngsters in their efforts to be more inclusive and to provide opportunities for autistics to have a better life quality in the society.

\section{Acknowledgment}

This study was supported with funding from Indonesian Endowment Fund for Education (LPDP) based at Jakarta, Indonesia as a scholarship funding for Ph.D. program at Curtin University, Western Australia.

\section{References}

[1] A. Tucker. "Interpreting and Treating Autism in Javanese Indonesia," ProQuest Dissertations Publishing, 2013.

[2] Y. E. Riany, M. Cuskelly, P. Meredith. "Cultural Beliefs about Autism in Indonesia," International Journal of Disability, Development and Education, Vol. 63, No. 6, pp. 623-640, 2016.

[3] Hersinta, "Tweeting Autism - A Framing Analysis of Twitter Conversations on Autism in Indonesia." in 4th International Conference on Contemporary Social and Political Affairs, Surabaya, Indonesia, A. Cashin, E. Abalos, A. Ismail, and P. Tunpungkom, Eds., 13 August 2018 2018, vol. 1: SciTePress, pp. 142-152, 2018.

[4] M. Mazurek. "Social media use among adults with autism spectrum disorders." Computers in Human Behavior, Vol. 29, No. 4, pp. 1709-1714, 2013.

[5] S. Neil, "The Meaning of Autism: Beyond Disorder." Disability \& Society, Vol. 23, No. 7, pp. 787-799, 2008.

[6] P. Jaarsma, S. Welin. "Autism as a natural human variation: reflections on the claims of the neurodiversity movement." Health Care Anal, Vol. 20, No. 1, pp. 20-30, 2012.

[7] A. Pinchevski. J. D. Peters. "Autism and new media: Disability between technology and society," New Media \& Society, July 8, 20152015.

[8] B. Penny, P. Standen. "The internet: a comfortable communication medium for people with Asperger syndrome (AS) and high functioning autism (HFA)?" Journal of Assistive Technologies, Vol. 3, No. 2, pp. 44-53, 2009.

[9] C. van der Aa, M. M. H. Pollmann, A. Plaat, R. J. van der Gaag. "Computer-mediated communication in adults with high-functioning autism spectrum disorders and controls." Research in Autism Spectrum Disorders, Vol. 23, pp. 15-27, 2016.

[10] M. Burke, R. Kraut, D. Williams. "Social use of computer-mediated communication by adults on the autism spectrum," presented at the Proceedings of the 2010 ACM conference on Computer supported cooperative work, Savannah, Georgia, USA, 2010.

[11] N. Bagatell. "Orchestrating voices: autism, identity and the power of discourse." Disability \& Society, Vol. 22, No. 4, pp. 413-426, 2007.

[12] M. Ward, R. Meyer. "Self-determination for people with developmental disabilities and autism: Two self-advocates' perspectives." Focus on Autism and Other Developmental Disabilities, Vol. 14, No. 3, p. 133, 1999. 
[13] Hersinta, "Different, not less: Communicating autism via the internet in Indonesia," in Interdisciplinary Approaches to Disability: Looking towards the Future, vol. 2, K. Ellis, R. Garland-Thomson, M. Kent, and R. Robertson Eds., pp. 219-229. New York: Routledge, 2019.

[14] B. A. Haller. Representing disability in an ableist world : essays on mass media. Louisville: The Advocado Press, 2010.

[15] M. Etty. Di Pintu Iman. Jakarta: PT Elex Media Komputindo, 2010.

[16] C. Thurlow. Computer Mediated Communication. London: SAGE Publications, 2004.

[17] J. Davidson. "Autistic culture online: virtual communication and cultural expression on the spectrum." Social \& Cultural Geography, Vol. 9, No. 7, pp. 791-806, 2008.

[18] J. Davidson, V. L. Henderson. "'Coming out' on the spectrum: autism, identity and disclosure," Social \& Cultural Geography, Vol. 11, No. 2, pp. 155-170, 2010.

[19] Departemen Kesehatan. Penyandang Disabilitas pada Anak. Retieved from: www.depkes.go.id/ download.php?file=download/...disabilitas.pdf, 2014.

[20] M. Gernsbacher, A. Raimond, M. Balinghasay, J. Boston. "'Special needs" is an ineffective euphemism," Principles and Implications, Vol. 1, No. 1, pp. 1-13, 2016.

[21] L. Wing. "The History of Ideas on Autism," Autism, Vol. 1, No. 1, pp. 13-23, 1997.

[22] H. U. Kim. "Autism across cultures: rethinking autism." Disability \& Society, Vol. 27, No. 4, pp. 535-545, 2012. 